\title{
WEIGHTED TRUDINGER - MOSER INEQUALITIES AND APPLICATIONS
}

\author{
M. Calanchi, The University of Milan, Milan, Italy, marta.calanchi@unimi.it, \\ B. Ruf, The University of Milan, Milan, Italy, bernhard.ruf@unimi.it
}

Trudinger - Moser inequalities provide continuous embeddings in the borderline cases of the standard Sobolev embeddings, in which the embeddings into Lebesgue $L^{p}$ spaces break down. One is led to consider their natural generalization, which are embeddings into Orlicz spaces with corresponding maximal growth functions which are of exponential type. Some parameters come up in the description of these growth functions. The parameter ranges for which embeddings exist increase by the use of weights in the Sobolev norm, and one is led to consider weighted TM inequalities. Some interesting cases are presented for special weights in dimension two, with applications to mean field equations of Liouville type.

Keywords: Trudinger - Moser inequalities; Orlicz spaces; maximal growth functions; weighted TM inequalities.

To the memory of Alfredo Lorenzi, with affection, admiration and gratitude.

Qual è 'l geomètra che tutto s'affige per misurar lo cerchio, e non ritrova, pensando, quel principio ond' elli indige, tal era io a quella vista nova: veder voleva come si convenne l'imago al cerchio e come vi s'indova

Dante Alighieri, Par. XXXIII 133-138

\section{Introduction}

\subsection{Why Trudinger - Moser Inequalities?}

For $\Omega \subset \mathbb{R}^{N}$ a bounded domain, the classical Sobolev embeddings are

$$
W_{0}^{1, p}(\Omega) \hookrightarrow L^{q}(\Omega) \quad \text { if } \quad p<N \quad \text { and } \quad 1 \leq q \leq \frac{p N}{N-p}=p^{*} .
$$

The Trudinger - Moser inequalities concern the borderline cases

$$
p=N, \text { for which formally } 1 \leq q \leq p^{*}=\infty,
$$

which leads to the question whether

$$
W_{0}^{1, p}(\Omega) \subset L^{\infty}(\Omega) .
$$

The answer is no, as simple examples show. The natural question then is to find the maximal growth function $\phi: \mathbb{R} \rightarrow \mathbb{R}^{+}$such that

$$
\text { if } u \in W_{0}^{1, N}(\Omega) \text {, then } \int_{\Omega} \phi(u) d x \text { is finite. }
$$


The answer was given by Yudovich [1], Peetre [2], Pohozaev [3] and Trudinger [4]: from their works one knows that $W_{0}^{1, N}(\Omega)$ embeds into the Orlicz space $L_{\phi}$, with growth function (so-called $N$-function) $\phi(t)=e^{|t|^{N-1}}-1$. This embedding is known by the name Trudinger inequality.

The proof is based on an expansion in power series of the exponential function, and on a control of the $L^{p}$ norm of each term of the series. This growth is optimal in the sense that for any higher growth there exist functions $u \in W_{0}^{1, N}(\Omega)$ for which the integral $\int_{\Omega} \phi(u) d x$ becomes infinite. Trudinger's inequality was made more precise by J. Moser [5], who showed that the supremum on the unit ball in $W_{0}^{1, N}(\Omega)$

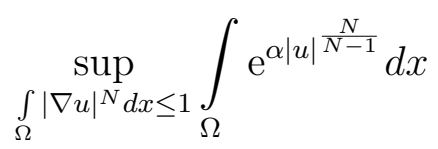

is bounded if and only if $\alpha \leq \alpha_{N}=N \omega^{\frac{1}{N-1}}$, where $\omega_{N-1}$ is the $N$-1-dimensional surface of the unit sphere. The integral is actually finite for any positive $\alpha$, but if $\alpha>\alpha_{N}$ it can be made arbitrarily large by a suitable choice of $u$.

From now on we will consider the two dimensional case. Let us recall Moser's result (known as the Trudinger - Moser inequality) in this case

Theorem 1. (Moser 1970 [5]). Let $N=2$; then

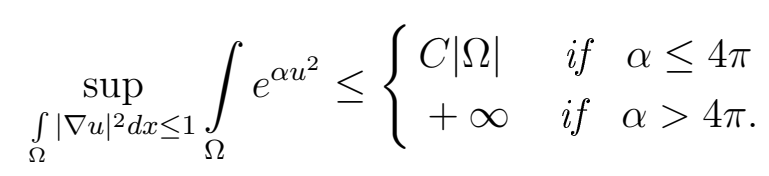

With a modification of the same arguments Moser also proved a corresponding inequality on the sphere $S^{2}$ which is useful for application in conformal deformation theory on manifolds.

Theorem 2. [5] If $u$ is a smooth function defined on the 2 dimensional sphere $S^{2}$ such that

$$
\int_{S^{2}}|\nabla u|^{2} d \mu \leq 1, \quad \int_{S^{2}} u d \mu=0,
$$

there exists a constant $C$ such that

$$
\int_{S^{2}} e^{4 \pi u^{2}} d \mu \leq C
$$

These inequalities are very useful to study 2-dimensional problems, such as the problem of prescribed Gauss curvature and mean field equation. In these contexts there is a more handy form.

\subsection{Logarithmic Trudinger - Moser Inequalities}

A variant of the TM inequality is the following logarithmic TM inequality: 
Theorem 3. Let $\Omega \subset \mathbb{R}^{2}$ be a bounded domain. Then there exists a constant $C>0$ such that

$$
\log \int_{\Omega} e^{u} d x \leq \frac{1}{16 \pi} \int_{\Omega}|\nabla u|^{2} d x+C, \quad u \in H_{0}^{1}(\Omega),
$$

while for the 2-dimensional unit sphere $S^{2}$ we have

$$
\frac{1}{2} \int_{S^{2}}|\nabla u|^{2} d x-8 \pi \log \int_{S^{2}} e^{u} d x \geq-C, \quad u \in H^{1}\left(S^{2}\right), \int_{S^{2}} u=0 .
$$

Proof. Using

$$
a b \leq \frac{a^{2}}{4 \varepsilon^{2}}+\varepsilon^{2} b^{2}
$$

applied to $b=\frac{u}{\|\nabla u\|_{2}}, a=\|\nabla u\|_{2}$ and $\varepsilon^{2}=4 \pi$, one has

$$
\begin{gathered}
\int_{\Omega} e^{u} d x \leq \int_{\Omega} e^{\frac{u}{\|\nabla u\|_{2}}\|\nabla u\|_{2}} d x \leq \int_{\Omega} e^{4 \pi \frac{u^{2}}{\|\nabla u\|_{2}^{2}}+\frac{1}{16 \pi}\|\nabla u\|_{2}^{2}} d x \leq \\
\leq e^{\frac{1}{16 \pi}\|\nabla u\|_{2}^{2}} \sup _{\|\nabla u\|_{2} \leq 1} \int_{\Omega} e^{4 \pi u^{2}} d x \leq C e^{\frac{1}{16 \pi}\|\nabla u\|_{2}^{2}}
\end{gathered}
$$

in virtue of the Trudinger - Moser inequality. Passing to the logarithm one obtains

$$
\log \int_{\Omega} e^{u} d x \leq \frac{1}{16 \pi}\|\nabla u\|_{2}^{2}+\log C
$$

so that (3) is proved.

The logarithmic TM inequality is crucial in the study of mean field equations of Liouville type (see [6]) of the form

$$
\left\{\begin{aligned}
-\Delta u & =\lambda \frac{e^{u}}{\int_{\Omega} e^{u}}, & & \text { in } \Omega \subset \mathbb{R}^{2} \\
u & =0 & & \text { on } \partial \Omega .
\end{aligned}\right.
$$

Equation (8) has been derived by Caglioti, Lions, Marchioro and Pulvirenti $[7,8]$ from the mean field limit of point vortices of the Euler flow, see also Chanillo - Kiessling [9] and Kiessling [10]. Equation (8) occurs also in the study of multiple condensate solutions for the Chern - Simons - Higgs theory, see Tarantello [11,12].

In particular, it has been shown (see e.g. $[13,14]$ ) that equation (8) has a solution which is a global minimizer of the associated energy functional if

$$
\lambda<8 \pi
$$

Here we concentrate our attention on some extensions of the previous results. 


\subsection{Weighted Trudinger - Moser Inequalities}

In some recent papers the influence of weights for such kind of inequalities has been studied.

In $[15,16]$ the authors consider the effect of power weights on the maximal growth in the integral term. In [17-19] the interest is devoted to the impact of weights in the Sobolev norm.

More precisely, let $w \in L^{1}(\Omega)$ be a non-negative function, and consider the weighted Sobolev space

$$
H_{0}^{1}(\Omega, w)=c l\left\{u \in C_{0}^{\infty}(\Omega) ; \int_{\Omega}|\nabla u|^{2} w(x) d x<\infty\right\} .
$$

For a general embedding theory for weighted Sobolev spaces, see Kufner [20].

It turns out that for weighted Sobolev spaces of form (10) logarithmic weights have a particular significance. However, as we will see below (Remark 2), we will need to restrict attention to radial functions to obtain interesting results. We are therefore led to consider problems of the following type: let $B \subset \mathbb{R}^{N}$ denote the unit ball in $\mathbb{R}^{N}$, and consider the weighted Sobolev space of radial functions

$$
H_{0, \text { rad }}^{1}(B, w)=c l\left\{u \in C_{0, \text { rad }}^{\infty}(B) ;\|u\|_{w}^{2}:=\int_{\Omega}|\nabla u|^{2} w(x) d x<\infty\right\}
$$

where

$$
w(x)=\left(\log \frac{1}{|x|}\right)^{\beta} \quad \text { or } \quad w(x)=\left(\log \frac{e}{|x|}\right)^{\beta}, \beta \geq 0 .
$$

Theorem 4. (Calanchi - Ruf, [17]) Let $\beta \in[0,1)$ and $w_{0}(x)=\left(\log \frac{1}{|x|}\right)^{\beta}$ or $w_{1}(x)=$ $\left(\log \frac{e}{|x|}\right)^{\beta}$. Then

(a) $\int_{B} e^{|u|^{\bar{\gamma}}} d x<+\infty$, for all $u \in H_{0, \text { rad }}^{1}(B, w) \Longleftrightarrow \bar{\gamma} \leq \gamma:=\frac{2}{1-\beta}$

and

$$
\sup _{\|u\|_{w} \leq 1, \operatorname{rad}} \int_{B} e^{\alpha|u|^{\gamma}} d x<+\infty
$$

if and only if

$$
\left.\alpha \leq \alpha_{\gamma}=2[2 \pi(1-\beta)]^{\frac{1}{1-\beta}}=2[2 \pi(2 / \gamma)]^{\gamma / 2} \quad \text { (critical growth }\right) .
$$

Remark 1. This result extends the Trudinger - Moser inequality; indeed, for $\beta=0$ we recover the classical TM inequality.

The proof is quite technical and we refer the reader to [17] for details. The idea is to transform the problem (which is one-dimensional by the radial symmetry) to an integral inequality on the half-line. In fact, by introducing the variable $t$ by $|x|=e^{-t / 2}$ and rescaling we obtain

$$
\psi(t)=2^{\frac{1-\beta}{2}}[2 \pi(1-\beta)]^{1 / 2} u(x), \quad \bar{\alpha}=\frac{\alpha}{2[2 \pi(1-\beta)]^{\frac{1}{1-\beta}}} .
$$


Then

$$
\int_{B}|\nabla u|^{2}|\log | x \|^{\beta} d x=\int_{0}^{\infty} \frac{\dot{\psi}^{2} t^{\beta}}{1-\beta} d t \text { and } \frac{1}{m(B)} \int_{B} e^{\alpha u^{\gamma}} d x=\int_{0}^{\infty} e^{\bar{\alpha} \psi^{\gamma}-t} d t .
$$

For the necessity of the conditions in $(b)$ it is sufficient to test

$$
\int_{0}^{\infty} e^{\bar{\alpha} \psi^{\gamma}-t} d t
$$

on the following Moser type functions

$$
\eta_{k}(t)= \begin{cases}\frac{t^{1-\beta}}{k^{\frac{1-\beta}{2}}} & 0 \leq t \leq k \\ k^{\frac{1-\beta}{2}} & t \geq k\end{cases}
$$

which satisfy $\left\|\eta_{k}\right\|_{w}=1$. If $\gamma=\frac{2}{1-\beta}$, then

$$
\int_{0}^{\infty} e^{\bar{\alpha} \eta_{k}^{\gamma}-t} d t \geq \int_{k}^{\infty} e^{\bar{\alpha} k-t} d t=e^{\bar{\alpha} k-k} \rightarrow \infty \text { if } \bar{\alpha}>1
$$

For the subcritical and critical cases, we need an estimate for functions $\psi$ which satisfy $\|\psi\|_{w}^{2}=\int_{0}^{\infty} \frac{\dot{\psi}^{2} t^{\beta}}{1-\beta} d t \leq 1$. In fact, by a radial lemma, see [17]), for such $\psi$ it holds that $|\psi(t)| \leq t^{1 / \gamma}$.

With this estimate the subcritical case can be trivially proved. If $\gamma \leq \frac{2}{1-\beta}$ and $\bar{\alpha}<1$, one easily obtains

$$
\int_{0}^{\infty} e^{\bar{\alpha} \psi^{\gamma}-t} d t \leq \int_{0}^{\infty} e^{\bar{\alpha} t-t} d t<\infty .
$$

As concerns the critical exponent (that is, the most delicate case) $\gamma=\frac{2}{1-\beta}$ and $\bar{\alpha}=1$, we follow an idea of Leckband [21], proving that the set of sublevels of the functions in the integral behaves well, and it is controlled by an integrable function on $[0,+\infty)$.

Going to the limiting case $\beta=1$ in Theorem 4, one has that the exponent $\gamma$ of $u$ in the integral goes to infinity, that is we are again in a borderline case. But again, the embedding does not go into $L^{\infty}$, but we find a critical growth of double exponential type, as described in the following

Theorem 5. [17] Let $w_{1}(x)=\left|\log \left(\frac{e}{|x|}\right)\right|($ i.e. $\beta=1)$. Then,

$$
\int_{B} e^{e^{|u|^{2}}} d x<+\infty \quad, \forall u \in H_{0, \operatorname{rad}}^{1}\left(B, w_{1}\right),
$$

while

$$
\sup _{\|u\|_{w_{1}} \leq 1, \text { rad }} \int_{B} e^{a e^{2 \pi u^{2}}} d x<+\infty \Longleftrightarrow a \leq 2 .
$$


Next, we consider the case $\beta>1$. In this case the functions in $H_{0, \text { rad }}^{1}\left(B, w_{1}\right)$ are bounded:

Theorem 6. [17] Let $\beta>1$. Then we have the following embedding

$$
H_{0, \text { rad }}^{1}\left(B, w_{1}\right) \hookrightarrow L^{\infty}(B)
$$

Also in these cases the idea is to reduce the problem to a one-dimensional integral inequality.

We conclude this first part with the following remark which explains why we have to restrict to radial functions.

Remark 2. If we do not restrict to radial function, that is, if we consider the whole weighted space $H_{0}^{1}(B, w)$, and the problem to find $\alpha, \beta, \gamma$ so that for $u \in H_{0}^{1}(B, w)$,

$$
\sup _{\|u\|_{w} \leq 1} \int_{B} e^{\alpha|u|^{\gamma}} d x<\infty,
$$

then we note that for $\gamma=2$ and $\alpha>4 \pi$ the supremum is not finite. Indeed, translating Moser's functions to a region in which the presence of the weight is not substantial (see [17]) one concludes that the presence of the weight has no effect for increasing the range of the inequality.

\subsection{Liouville Type Equations with Logarithmic Coefficients}

In this article we will derive some new logarithmic Sobolev inequalities with weights, and will then apply them to prove existence results for associated mean field equations of Liouville type, with elliptic operators with logarithmic coefficients. In particular, we will prove

Theorem 7. Let $w=w(x)=\left(\log \frac{e}{|x|}\right)^{\beta}$ be a logarithmic weight, with $\beta>0$. Then, for any $\lambda>0$, the equation

$$
\left\{\begin{aligned}
-\operatorname{div}(w(x) \nabla u) & =\lambda \frac{e^{u}}{\int_{B} e^{u}}+f(x) & & \text { in } B \\
u & =0 & & \text { on } \partial B
\end{aligned}\right.
$$

has a (weak) radial solution $u \in H_{0}^{1}(B, w)$ which is a global minimizer of the associated energy functional.

We recall that for the corresponding equation with $\beta=0$ there exists a global minimizer solution only for $\lambda$ in the range

$$
\lambda<8 \pi \text {. }
$$

This is due to the fact that for $\beta>0$ the growth $e^{u}$ is not the maximal possible. If we increase the growth to critical growth $e^{|u|^{\gamma}}$, then we find an analogous restriction to (13) to obtain a global minimizer for the associated equation: 
Theorem 8. Let $w(x)=\left(\log \frac{e}{|x|}\right)^{\beta}$. Then, with $\theta=\frac{2 \gamma}{2+\gamma}$, the equation

$$
\left\{\begin{aligned}
-\operatorname{div}(w(x) \nabla u) & =\lambda \frac{|u|^{\theta-2} u e^{|u|^{\theta}}}{\int_{B} e^{|u|^{\theta}}}+f(x) & & \text { in } B \\
u & =0 & & \text { on } \partial B
\end{aligned}\right.
$$

has a weak radial solution, which is a global minimizer of the associated energy functional, for every $\lambda$ satisfying

$$
\lambda<\frac{2 \pi}{\gamma^{2}}(2+\gamma)^{\frac{2+\gamma}{\gamma}}
$$

Note that for $\theta=1$, i.e. $\gamma=2$, the condition (15) reduces to $\lambda<8 \pi$, which is the condition (9) of the mean field Liouville equation.

Finally, we prove an existence theorem for the following elliptic mean field equation with a double exponential growth

Theorem 9. Let $w=\log \frac{e}{|x|}$. Then the equation

$$
\left\{\begin{aligned}
-\operatorname{div}\left(\log \frac{e}{|x|} \nabla u\right) & =\lambda \frac{e^{u}}{\log \int_{B} e^{e^{u}}} \frac{e^{e^{u}}}{\int_{B} e^{e^{u}}}+f(x) & & \text { in } B \\
u & =0 & & \text { on } \partial B
\end{aligned}\right.
$$

has a global radial minimizer solution for every

$$
\lambda<\pi \text {. }
$$

\section{Weighted Logarithmic TM Inequalities}

In this section we prove some logarithmic TM inequalities with weights. We start with a simple inequality based on the previous weighted TM embeddings, and apply it to prove Theorem 7 .

Proposition 1. Let $w(x)=\left(\log \frac{e}{|x|}\right)^{\beta}$ with $\beta>0$. Then for every $\lambda>0$ there exists a constant $C=C(\lambda, \beta)$ which depends on $\lambda$ and $\beta$ such that

$$
\frac{1}{2}\|u\|_{w}^{2}-\lambda \log \int_{B} e^{u} \geq \frac{1}{4}\|u\|_{w}^{2}-C
$$

where

$$
\|u\|_{w}^{2}=\int_{B}|\nabla u|^{2} w(x) d x
$$

Proof. Let $\beta \in(0,1)$. Reasoning as in Remark 1, using again inequality (5), we have

$$
\log \int_{B} e^{u} \leq \log \left(\int_{B} e^{\frac{1}{4 \varepsilon^{2}}\left(\frac{|u|}{\|u\|_{w}}\right)^{2}+\varepsilon^{2}\|u\|_{w}^{2}} d x\right) \leq
$$




$$
\leq \varepsilon^{2}\|u\|_{w}^{2}+\log \left(\int_{B} e^{\frac{1}{4 \varepsilon^{2}}\left(\frac{|u|}{\|u\|_{w}}\right)^{2}} d x\right)
$$

Now we choose $\varepsilon^{2}=\frac{1}{4 \lambda}$. There exists a constant $C_{1}=C_{1}(\gamma, \lambda)$ which depends only on $\varepsilon$ (hence on $\lambda$ ) and $\gamma=\frac{2}{1-\beta}>2$, such that

$$
\int_{B} e^{\frac{1}{4 \varepsilon^{2}}\left(\frac{|u|}{\|u\|_{w}}\right)^{2}} d x \leq \int_{B} e^{C_{1}+\alpha_{\gamma}\left(\frac{|u|}{\|u\|_{w}}\right)^{\gamma}} d x
$$

here $\alpha_{\gamma}$ is the critical exponent given in Theorem 4. By (b) of the same theorem we have that

$$
\begin{array}{r}
\log \left(\int_{B} e^{\frac{1}{4 \varepsilon^{2}}\left(\frac{|u|}{\|u\|_{w}}\right)^{2}} d x\right) \leq \log \int_{B} e^{C_{1}+\alpha_{\gamma}\left(\frac{|u|}{\|u\|_{w}}\right)^{\gamma}} d x \\
\leq C_{1}(\gamma, \lambda)+\log \int_{B} e^{\alpha_{\gamma}\left(\frac{|u|}{\|u\|_{w}}\right)^{\gamma}} d x \leq C .
\end{array}
$$

With a similar argument (using Theorem 5 and Theorem 6) one can prove the result in the case $\beta \geq 1$.

\section{Applications to Liouville Type Equations}

Consider the following elliptic boundary value problem

$$
\left\{\begin{aligned}
-\operatorname{div}(w(x) \nabla u) & =\lambda \frac{e^{u}}{\int_{B} e^{u}}+f & & \text { in } B \\
& u=0 & & \text { on } \partial B
\end{aligned}\right.
$$

where $w=w(x)=\left(\log \frac{e}{|x|}\right)^{\beta}$ is a logarithmic weight with $\beta>0$, and $f \in L^{2}(B)$.

We look for weak solutions of (LP), i.e. a function $u \in H_{0, \text { rad }}^{1}(B, w)$ satisfying

$$
\int_{B} \nabla u \nabla v w(x) d x=\lambda \frac{\int_{B} e^{u} v d x}{\int_{B} e^{u} d x}+\int_{B} f v d x, \quad \forall v \in C_{0, \text { rad }}^{\infty}(B),
$$

i.e. $u$ is a weak solution if and only if it is a critical point of the functional $J$ : $H_{o, \text { rad }}^{1}(B, w) \rightarrow \mathbb{R}$ associated to equation (19) and given by

$$
J(u)=\frac{1}{2}\|u\|^{2}-\lambda \log \int_{B} e^{u} d x-\int_{B} f u d x .
$$

Вестник ЮУрГУ. Серия «Математическое моделирование 
Proposition 2. For every $\lambda>0$ problem (19) has a weak radial solution which corresponds to the minimum of the functional $J(u)$.

Proof. We first prove that $J$ is coercive for every $\lambda$ fixed. Indeed by Proposition 1, one has

$$
J(u) \geq \frac{1}{4}\|u\|_{w}^{2}-C-D\|u\|_{w}\|f\|_{2}
$$

for a suitable constant $D$. Then $J$ is bounded from below.

Let

$$
m=\inf _{u \in \tilde{H}} J(u)
$$

and let $\left\{u_{n}\right\}_{n}$ be a minimizing sequence, i.e.

$$
J\left(u_{n}\right) \rightarrow m, \text { as } n \rightarrow+\infty .
$$

By the coercivity of $J, u_{n}$ is bounded in $H_{0, \text { rad }}^{1}(B, w)$. Therefore there exists $u \in$ $H_{0, \text { rad }}^{1}(B, w)$, such that (up to a subsequence)

$$
u_{n} \rightarrow u \text { in } H_{0, r a d}^{1}(B, w), u_{n} \rightarrow u \text { in } L^{1}(B), u_{n} \rightarrow u \text { a.e. }, \text { as } n \rightarrow+\infty \text {. }
$$

Observe that the nonlinearity $e^{u}$ is subcritical and that there exists a constant $C_{1}$ such that

$$
\left|t e^{t}\right| \leq C_{1} e^{\alpha_{\gamma}|t|^{\gamma}}
$$

From Theorem 4 and this estimate we have that

$$
e^{u_{n}} \in L^{1} \text { and } \int_{B}\left|e^{u_{n}} u_{n}\right| d x \leq C .
$$

We now apply the following result due to de Figueiredo - Miyagaki - Ruf [22, lemma 2.1].

Lemma 1. Let $\left(u_{n}\right)_{n}$ be a sequence of functions in $L^{1}(\Omega)$ converging to $u$ in $L^{1}(\Omega)$. Assume that $F\left(u_{n}(x)\right)$ and $F(u(x))$ are also $L^{1}$ functions. If

$$
\int_{\Omega}\left|F\left(u_{n}(x)\right) u_{n}(x)\right| \leq C
$$

then $F\left(u_{n}(x)\right)$ converges to $F(u(x))$ in $L^{1}$.

We then obtain, for $F(t)=e^{t}$

$$
\int_{B} e^{u_{n}} \rightarrow \int_{B} e^{u}, \text { as } n \rightarrow+\infty,
$$

and by lower semi-continuity of the norm $\|\cdot\|_{w}^{2}$

$$
m \leq J(u) \leq \liminf _{n \rightarrow+\infty} J\left(u_{n}\right)=m
$$

So that $u$ is a global minimum (and then a critical point) of $J$.

This completes also the proof of Theorem 7 . 


\section{A Critical Weighted TM Inequality}

Let $w(x)=\left(\log \frac{1}{|x|}\right)^{\beta}$ the logarithmic weight with $\beta \in(0,1)$.

Consider the functional $J_{\lambda}: H_{0, \text { rad }}^{1}(B, w) \rightarrow \mathbb{R}$ defined as follows

$$
J_{\lambda}(u):=\frac{1}{2}\|u\|_{w}^{2}-\lambda \log \left(\int_{B} e^{|u|^{\theta}} d x\right)-\int_{B} f u d x
$$

where $\|u\|_{w}^{2}=\int_{B}|\nabla u(x)|^{2} w(x) d x, \theta=\frac{2 \gamma}{2+\gamma}$ ( $\gamma$ is the critical growth given by (b), Theorem $4)$, and $f \in L^{2}(B)$.

Proposition 3. $J_{\lambda}$ is coercive for all $\lambda<\frac{2 \pi}{\gamma^{2}}(2+\gamma)^{\frac{2+\gamma}{\gamma}}$.

We need a preliminary inequality

Lemma 2. Let $\delta$, $\delta^{\prime}$ two conjugate exponents. For every $s, t \geq 0$ one has

$$
s t \leq \frac{(\alpha s)^{\delta^{\prime}}}{\delta^{\prime}}+\frac{t^{\delta}}{\delta \alpha^{\delta}}, \quad \forall \alpha>0
$$

Proof. This is a classical convexity inequality, which can be deduced by Jensen's inequality. Here we give an alternative proof. In fact, considering

$$
\sup _{s \geq 0}\left\{s t-\frac{1}{p} s^{p}\right\}
$$

we get, by setting the derivative equal zero in the maximum point, $t=s^{p-1}$, hence $s(t)=$ $t^{1 /(p-1)}$, and then

$$
\begin{gathered}
\sup _{s \geq 0}\left\{s t-\frac{1}{p} s^{p}\right\}=t^{1 /(p-1)} t-\frac{1}{p} t^{p /(p-1)} \\
=\left(1-\frac{1}{p}\right) t t^{p /(p-1)}=\frac{1}{q} t^{q} .
\end{gathered}
$$

To obtain other constants in the inequality, it is sufficient to consider

$$
(\alpha s)\left(\frac{1}{\alpha} t\right) \leq \frac{(\alpha s)^{\delta^{\prime}}}{\delta^{\prime}}+\frac{t^{\delta}}{\delta \alpha^{\delta}}, \quad \forall \alpha>0 .
$$

Proof. (of Proposition 3) We first prove that

$$
\log \int_{B} e^{|u|^{\theta}} d x \leq \frac{\|u\|_{w}^{2}}{A(\gamma)}+\log \left(\int_{B} e^{\alpha_{\gamma}\left(\frac{|u|}{\|u\|_{w}}\right)^{\gamma}} d x\right),
$$

where $A(\gamma)=\frac{4 \pi}{\gamma^{2}}(2+\gamma)^{\frac{2+\gamma}{\gamma}}$ and $\alpha_{\gamma}=2[2 \pi(2 / \gamma)]^{\gamma / 2}$ is the critical exponent given in Theorem 4 (notice that $A(2)=16 \pi$ and $\alpha_{2}=4 \pi$ gives the classical TM inequality for the Liouville problem). 
We use inequality (22), where $\delta, \delta^{\prime}$ are conjugate exponents, such that

$$
\left\{\begin{array} { l } 
{ \theta \delta = \gamma } \\
{ \theta \delta ^ { \prime } = 2 }
\end{array} \quad \text { that is } \quad \left\{\begin{array}{l}
\delta=\frac{2+\gamma}{2} \\
\delta^{\prime}=\frac{2+\gamma}{\gamma}
\end{array}\right.\right.
$$

One has, by taking $s=\|u\|_{w}^{\theta}$ and $t=\left(\frac{|u|}{\|u\|_{w}}\right)^{\theta}$ in (22),

$$
|u|^{\theta} \leq \alpha_{\gamma}\left(\frac{|u|}{\|u\|_{w}}\right)^{\gamma}+\frac{\|u\|_{w}^{2}}{A(\gamma)}
$$

and the first inequality is proven.

Let

$$
C=\sup _{\|u\|_{w} \leq 1, u \in \tilde{H}} \int_{B} e^{\alpha_{\gamma}\left(\frac{|u|}{\|u\|_{w}}\right)^{\gamma}} d x
$$

Then

$$
\begin{aligned}
J_{\lambda}(u):=\frac{1}{2}\|u\|_{w}^{2} & -\lambda \log \left(\int_{B} e^{|u|^{\theta}} d x\right)-\int_{B} f u d x \\
& \geq\left(\frac{1}{2}-\frac{\lambda}{A(\gamma)}\right)\|u\|_{w}^{2}-\lambda \log C-D\|f\|_{2}\|u\|_{w}
\end{aligned}
$$

and the proof is complete.

Let us now consider the following Liouville-type equation

$$
\left\{\begin{aligned}
-\operatorname{div}(w(x) \nabla u) & =\lambda \frac{|u|^{\theta-2} u e^{|u|^{\theta}}}{\int_{B} e^{|u|^{\theta}}}+f & & \text { in } B \\
u & =0 & & \text { on } \partial B
\end{aligned}\right.
$$

Again by the coercivity of the functional $J_{\lambda}$ one can prove the following existence result

Proposition 4. For every $\lambda<\frac{2 \pi}{\gamma^{2}}(2+\gamma)^{\frac{2+\gamma}{\gamma}}$ the functional $J_{\lambda}$ attains its minimum, and therefore problem (23) possesses a weak radial solution.

The proof is very similar to that of Theorem 7 . This completes the proof of Theorem 8 .

\section{A Double Exponential Weighted Liouville Type Problem}

Consider the functional $I: H_{0}^{1}(B, w) \rightarrow \mathbb{R}$ defined as follows

$$
I_{\lambda}(u):=\frac{1}{2}\|u\|_{\log }^{2}-\lambda \log \log \left(\int_{B} e^{e^{u}} d x\right)
$$

where $\|u\|_{\log }^{2}=\int_{B}|\nabla u(x)|^{2}\left(\log \frac{e}{|x|}\right) d x$. We have the following $\log \log$-inequality: 
Proposition 5. It holds

$$
\log \log \left(\int_{B} e^{e^{u}} d x\right) \leq \frac{1}{2 \pi}\|u\|_{\log }^{2}+\log \left(\frac{1}{8}+\frac{\log C_{M B}}{e^{\frac{\|u\|_{\log }^{2}}{2 \pi}}}\right) \quad \forall u \in H_{0}^{1}(B, w)
$$

Therefore the functional $I_{\lambda}$ is coercive for every $\lambda<\pi$ and it has an absolute minimum.

Proof.Taking $a=\|u\|_{w}, b=\frac{|u|}{\|u\|_{w}}$ and $\varepsilon^{2}=\pi$ in

$$
a b \leq \frac{a^{2}}{4 \varepsilon^{2}}+\varepsilon^{2} b^{2}
$$

we have

$$
|u| \leq \frac{1}{4 \pi}\|u\|_{w}^{2}+\pi\left(\frac{u}{\|u\|_{w}}\right)^{2}
$$

so that

$$
\log \log \left(\int_{B} e^{e^{u}} d x\right) \leq \log \log \int_{B} e^{e^{\frac{1}{8 \pi}\|u\|_{w}^{2}+2 \pi\left(\frac{u}{\|u\|_{w}}\right)^{2}}} d x=\log \log \int_{B} e^{e^{\frac{1}{4 \pi}\|u\|_{w}^{2}} e^{\pi\left(\frac{u}{\|u\|_{w}}\right)^{2}}} d x
$$

Let

$$
C_{M B}=\sup _{\|u\|_{w} \leq 1, \mathrm{rad}} \int_{B_{1}(0)} e^{2 e^{2 \pi|u|^{2}}} d x
$$

(which is finite in virtue of Theorem 5).

Now taking $a=e^{\frac{1}{4 \pi}\|u\|_{w}^{2}}, b=e^{\pi\left(\frac{u}{\|u\|_{w}}\right)^{2}}$ and $\varepsilon^{2}=2$ in the inequality above, one gets

$$
\begin{gathered}
\log \log \left(\int_{B} e^{e^{u}} d x\right) \leq \log \log \int_{B} e^{\frac{1}{8} e^{\frac{1}{2 \pi}\|u\|_{w}^{2}}+2 e^{2 \pi\left(\frac{u}{\|u\|_{w}}\right)^{2}} d x=} \\
=\log \left(\log \int_{B} e^{2 e^{2 \pi\left(\frac{u}{\|u\|_{w}}\right)^{2}}} d x+\frac{1}{8} e^{\frac{1}{2 \pi}\|u\|_{w}^{2}}\right) \leq \\
\leq \log \left(\log C_{M B}+\frac{1}{8} e^{\frac{1}{2 \pi}\|u\|_{w}^{2}}\right) \leq \frac{1}{2 \pi}\|u\|_{w}^{2}+\log \left(\frac{1}{8}+\frac{\log C_{M B}}{e^{\frac{\|u\|_{w}}{2 \pi}}}\right) .
\end{gathered}
$$

Moreover

$$
I_{\lambda}(u) \geq\left(\frac{1}{2}-\frac{\lambda}{2 \pi}\right)\|u\|_{w}^{2}-\lambda \log \left(\frac{1}{8}+\frac{\log C_{M B}}{e^{\frac{\|u\|_{w}^{2}}{2 \pi}}}\right) .
$$

For $\lambda<\pi$ the functional (and every perturbation with a linear term) is coercive, therefore it is bounded from below. In order to prove the existence of a minimum it is sufficient to argue as in Theorem 2. 
Finally we point out that any minimizer of $I_{\lambda}$ satisfies the following elliptic problem with a double exponential growth.

$$
\left\{\begin{aligned}
-\operatorname{div}\left(\log \frac{e}{|x|} \nabla u\right) & =\lambda \frac{e^{u}}{\log \int_{B} e^{e^{u}}} \frac{e^{e^{u}}}{\int_{B} e^{e^{u}}}+f & & \text { in } B \\
u & =0 & & \text { on } \partial B
\end{aligned}\right.
$$

and hence the proof of Theorem 9 is complete.

\section{References}

1. Yudovich V.I. Some Estimates Connected with Integral Operators and with Solutions of Elliptic Equations. Dokl. Akad. Nauk SSSR, 1961, vol. 138, pp. 805-808.

2. Peetre J. Espaces d' interpolation et théorème de Soboleff. Ann. Inst. Fourier, 1966, vol. 16, pp. 279-317. DOI: 10.5802 /aif.232

3. Pohozaev S.I. The Sobolev Embedding in the Case $p l=n$. Proc. of the Technical Scientific Conference on Advances of Scientific Research, 1964 - 1965, Mathematics Section, (Moskov. Energet. Inst., Moscow), pp. 158-170.

4. Trudinger N.S. On Imbeddings into Orlicz Spaces and Some Applications. Journal of Applied Mathematics and Mechanics, 1967, vol. 17, pp. 473-483. DOI: 10.1512/iumj.1968.17.17028

5. Moser J. Moser J. A Sharp Form of an Inequality by N. Trudinger. Indiana University Mathematics Journal, 1970/71, vol. 20, pp. 1077-1092. DOI: 10.1512/iumj.1971.20.20101

6. Liouville J., Sur l' equation aux derivées partielles. Journal de Mathématiques Pures et Appliquées, 1853, vol. 18, pp. 71-72.

7. Caglioti E., Lions P.L., Marchioro C., Pulvirenti M. A Special Class of Stationary Flows for Two-Dimensional Euler Equations: a Statistical Mechanics Description. Communications in Mathematical Physics, 1992, vol. 143, no. 3, pp. 501-525. DOI: 10.1007/BF02099262

8. Caglioti E., Lions P.L., Marchioro C., Pulvirenti M. A Special Class of Stationary Flows for Two-Dimensional Euler Equations: a Statistical Mechanics Description. II. Communications in Mathematical Physics, 1995, vol. 174, no. 2, pp. 229-260. DOI: 10.1007/BF02099602

9. Chanillo S., Kiessling M. Rotational Symmetry of Solutions of Some Nonlinear Problems in Statistical Mechanics and in Geometry. Communications in Mathematical Physics, 1994, vol. 160, no. 2, pp. 217-238. DOI: 10.1007/BF02103274

10. Kiessling M.K.-H. Statistical Mechanics of Classical Particles with Logarithmic Interactions. Communications on Pure and Applied Mathematics, 1993, vol. 46, pp. 27-56. DOI: $10.1002 /$ cpa.3160460103

11. Tarantello G. Multiple Condensate Solutions for the Chern - Simons - Higgs Theory. Journal of Mathematical Physics, 1996, vol. 37, pp. 3769-3796. DOI: 10.1063/1.531601

12. Tarantello G. Analytical Aspects of Liouville-Type Equations with Singular Sources. Handbook of Differential Equations (M. Chipot and P. Quittner, eds.), Elsevier, North Holland, 2004, pp. 491-592.

13. Li Y.Y. Harnack Type Inequality: the Method of Moving Planes. Communications in Mathematical Physics, 1999, vol. 200, pp. 421-444. DOI: 10.1007/s002200050536

14. Chen C.C., Lin C.S. Mean Field Equations of Liouville Type with Singular Data: Sharper Estimates. Discrete and Continuous Dynamic Systems, 2010, vol. 28, no. 3, pp. 123-127. 
15. Calanchi M., Terraneo E. Non-radial Maximizers for Functionals with Exponential Nonlinearity in $\mathbb{R}^{2}$. Advanced Nonlinear Studies, 2005, vol. 5, pp. 337-350.

16. Adimurthi, Sandeep K. A Singular Moser - Trudinger Embedding and Its Applications. Nonlinear Differential Equations and Applications, 2007, vol. 13, issue 5-6, pp. 585-603. DOI: $10.1007 / \mathrm{s} 00030-006-4025-9$

17. Calanchi M., Ruf B. On a Trudinger - Moser Type Inequality with Logarithmic Weights. Journal of Differential Equations, 2015, no. 3, pp. 258-263. DOI: 10.1016/j.jde.2014.11.019

18. Calanchi M. Some Weighted Inequalitie of Trudinger - Moser Type in Progress. Nonlinear Differential Equations and Applications, Birkhauser, 2014, vol. 85, pp. 163-174.

19. Calanchi M., Ruf B. Trudinger - Moser Type Inequalities with Logarithmic Weights in Dimension N. Nonlinear Anal., 2015, vol. 121, pp. 403-411.

20. Kufner A. Weighted Sobolev Spaces. John Wiley \& Sons Ltd, 1985.

21. Leckband M.A. An Integral Inequality with Applications. Transactions of the American Mathematical Society, 1984, vol. 283, no. 1, pp. 157-168. DOI: 10.1090/S0002-9947-19840735413-7

22. de Figueiredo G., Miyagaki O.H., Ruf B. Elliptic Equations in $\mathbb{R}^{2}$ with Nonlinearities in the Critical Growth Range. Calc. Var. Partial Differential Equations, 1995, vol. 3, no. 2, pp. 139-153. DOI: 10.1007/BF01205003

Received January 24, 2015

УДК 517.954, 517.96

DOI: $10.14529 / \mathbf{m m p} 150303$

\title{
ВЗВЕШАННЫЕ НЕРАВЕНСТВА ТРУДИНГЕРА - МОЗЕРА И ПРИЛОЖЕНИЯ
}

\author{
М. Каланки, Б. Руф
}

\begin{abstract}
Неравенства Трудингера - Мозера обеспечивают непрерывные вложения в пограничных случаях стандартных вложенияй Соболева, в которых вложения в пространствах Лебега $L^{p}$ отсутствуют. В этом случае приходится рассматривать их естественные обобщения, которые являются вложениями в пространства Орлича соответствующих функциям максимального роста, экспоненциального типа. При описании этих функций роста возникают некоторые параметры. Диапазоны параметров, для которых существуют вложения, можно увеличить с помощью введения весов в нормах Соболева, что и приводит к рассмотрению весовых неравенств ТМ. Представлены некоторые интересные случаи со специальными весами в двумерной области, с приложением к уравнениям среднего поля типа Лиувилля.

Ключевые слова: неравенства Трудингера - Мозера; пространства Орлича; максимальные функции роста; весовые неравенства ТМ.
\end{abstract}

Марта Каланки, кафедра математики, Миланский университет, Милан, Италия, marta.calanchi@unimi.it.

Бернард Руф, кафедра математики, Миланский университет, Милан, Италия, bernhard.ruf@unimi.it

Поступила в редакиию 24 января 2015 г. 